\title{
Développement des caractéristiques biochimiques et sensorielles des fromages de chèvre. Étude interespèce par échange de la matière grasse et du lait écrémé de laits de vache et de chèvre
}

\author{
Solange Buchin ${ }^{\text {**, Gabriel Duboz }}{ }^{\mathrm{a}}$, Jean-Luc Le Quéréb, \\ Rémy Grappin ${ }^{\mathrm{a}}$
}
a Station de recherches en technologie et analyses laitières, Inra, BP 89, 39801 Poligny cedex, France
${ }^{b}$ Laboratoire de recherches sur les arômes, Inra, 17, rue Sully, BV 1540, 21034 Dijon cedex, France

(Reçu le 7 octobre 1997 ; accepté le 30 juin 1998)

\begin{abstract}
Development of biochemical and sensory characteristics of goat cheese. Interspecies study by exchange of the fatty fraction and the skimmed fraction of cow's and goat's milk. Soft and semi-hard cheeses were manufactured with goat's and cow's milk, either with the original milk, or after exchange of the skimmed and the fatty fractions. The physico-chemical and sensory characteristics of the four types of cheeses obtained by both technologies were analyzed. Within the semi-hard cheeses group, more proteolytic activity was observed in goat's skimmed milk cheeses. In both soft and semi-hard cheeses, cow's milk produced more lipolyzed cheeses. The goaty flavour of semi-hard cheeses was strongly related to the presence of goat's fatty fraction, whereas in the soft cheeses, the goaty flavour was influenced by both milk fractions. The perception of this flavour in the cheeses was related to the presence of C6:0, C8:0, C9:0, C10:0, 4MeC8:0 and 4EtC8:0 fatty acids. Milky and buttery odours were related to cow's milk fat, whereas pungent, rancid, acid and bitter attributes differed between soft and semi-hard cheeses. The texture characteristics were only influenced by the skimmed milk fraction in semi-hard cheeses, whereas they were also related to the fatty fraction in soft cheeses. (c) Inra/Elsevier, Paris.
\end{abstract}

goat's cheese / proteolysis / lipolysis / volatile compound / flavour / texture

Résumé - Des fromages à pâte molle et à pâte pressée ont été fabriqués à partir de lait de chèvre et de vache, soit en conservant les laits d'origine, soit en échangeant les fractions grasse et écrémée. Les caractéristiques physico-chimiques et sensorielles des quatre types de fromages obtenus pour chaque technologie ont été analysées. Dans les pâtes pressées, les fromages à lait écrémé de chèvre sont

\footnotetext{
* Correspondance et tirés à part. E-mail : buchin@ poligny.inra.fr
} 
plus protéolysés. Dans les deux technologies, le lait de vache donne des fromages plus lipolysés. L'arôme chèvre des fromages à pâte pressée est fortement lié à la présence de matière grasse de chèvre, alors que dans les fromages à pâte molle, il est influencé par les deux fractions du lait. La perception de cet arôme dans les fromages est en relation avec la présence des acides gras C6:0, C8:0, C9:0, C10:0, 4MeC8:0 et 4EtC8:0. Les odeurs de lait et de beurre sont liées à la présence de matière grasse de vache, alors que la répartition entre fromages des caractères piquant, rance, acide et amer diffère selon la technologie. La texture, uniquement influencée par la fraction écrémée dans les fromages à pâte pressée, est aussi liée à la fraction grasse dans les fromages à pâte molle. (C) Inra/Elsevier, Paris.

\section{fromage de chèvre / protéolyse / lipolyse / composé volatil / flaveur / texture}

\section{INTRODUCTION}

Plusieurs travaux ont été entrepris ces dernières années afin de déterminer les facteurs intervenant sur les caractéristiques sensorielles des fromages de chèvre, par la comparaison de laits de chèvre de différents variants génétiques de la caséine $\alpha_{\mathrm{s} 1}$ ou de fromages fabriqués avec ces laits $[8,17,19$, $29,30,32$ ]. Il est ainsi apparu que la fermeté des fromages est corrélée à l'extrait $\mathrm{sec}$, au gras / $\mathrm{sec}$, et au $\mathrm{pH}[8,32]$, et l'arôme « chèvre » essentiellement à l'acidité grasse et à l'activité lipasique des laits [8, 19, 29].

Une meilleure compréhension des différences sensorielles observées en fonction du type de lait peut être obtenue en faisant la part de la matière grasse et de la fraction écrémée dans ce phénomène. En effet, si la texture des fromages est connue pour dépendre étroitement de la structure des micelles de caséine et de la protéolyse [20], il semble que la matière grasse ait également un rôle non négligeable sur la texture des fromages, en particulier en raison de son pouvoir lubrifiant [26]. En ce qui concerne l'arôme des fromages, il résulte d'un équilibre entre de nombreuses molécules aromatiques, d'origine aussi bien lipidique que protéique ou fermentaire [1]. La plupart de ces molécules possèdent un arôme atypique, bien que certaines semblent plus spécifiques du fromage. C'est le cas par exemple de certaines méthyl-cétones à l'arôme de fromage bleu, de certains acides gras à l' arôme de fromage [31], ou d'acides gras, en particulier des acides ramifiés, qui présentent un arôme « chèvre » $[4,23]$. Dans ce but, l'étude de Lamberet et al. [19], qui a consisté à échanger la matière grasse et les protéines provenant de laits de chèvre de différents variants génétiques de la caséine $\alpha_{s 1}$ pour la fabrication de fromages, a montré que la fermeté des fromages est associée à la fraction protéique, alors que les fractions grasse et protéique interviendraient toutes deux dans l'arôme " chèvre ».

Le même objectif nous a conduit, dans la présente étude, à réaliser également un échange de matière grasse et de protéines, mais à partir de laits plus différents. Le choix a porté sur une comparaison inter-espèce de laits de chèvre et de vache, qui présentent de grandes différences de compositions en caséines et en acides gras. Le lait de chèvre contient une proportion plus faible de caséine $\alpha_{s 1}$ que le lait de vache, et des proportions plus élevées d'acides caproïque, caprylique, caprique et laurique. Par ailleurs, la taille des micelles de caséine et des globules gras est différente [21]. Une approche similaire a déjà été réalisée par Banks et al. [3] sur du cheddar, avec une comparaison de laits de vache et de brebis.

Le schéma expérimental a consisté à séparer les fractions écrémée et grasse de chaque type de lait et à les recombiner de façon à obtenir deux laits pur vache ou pur chèvre, et deux laits de mélange. À partir 
des quatre types de laits obtenus, des fromages ont été fabriqués selon deux technologies: une technologie pâte molle de type Sainte Maure et une technologie pâte pressée de type Saint Paulin qui permet de s'affranchir de l'influence éventuelle de la flore de surface dont les activités métaboliques peuvent masquer des différences dues à la matière première $[8,17]$. Les caractéristiques sensorielles de ces fromages ont été mises en relation avec des phénomènes biochimiques tels que la protéolyse, la lipolyse ou la formation de composés volatils.

\section{MATÉRIEL ET MÉTHODES}

\subsection{Protocole expérimental}

Dans cette étude, le lait de vache provenait d'un lait de grand mélange de vaches de race Montbéliarde et le lait de chèvre d'un troupeau de 15 chèvres de race Alpine. Les fabrications de fromages à pâte pressée type mini Saint-Paulin $\mathrm{PP})$ et fromages à pâte molle type Sainte Maure (PM) ont été réalisées lorsque les chèvres étaient en milieu de lactation (mois de juillet). Trente litres de chaque lait ont été utilisés pour chaque fabrication. Après un écrémage total du lait (les laits écrémés de chèvre contenaient de 0,3 à $1,0 \mathrm{~g} \cdot \mathrm{L}^{-1}$ de matière grasse résiduelle, ceux de vache de 0,7 à $\left.1,7 \mathrm{~g} \cdot \mathrm{L}^{-1}\right)$, les fractions ont été mélangées avec standardisation du rapport taux protéique / taux butyreux de 0,95 à 1,00 pour les $\mathrm{PP}$, et de 1,20 pour les PM, selon quatre combinaisons :

$\begin{array}{lll} & \text { Crème vache Crème chèvre } \\ \text { Lait écrémé vache VV } & \text { VC } \\ \text { Lait écrémé chèvre CV } & \text { CC }\end{array}$

Ces codes seront utilisés par la suite pour désigner les types de lait utilisés, la première lettre désignant l'origine de la fraction écrémée, la deuxième celle de la matière grasse. Pour chaque type de fromages, les fabrications ont été répétées quatre fois au cours d'une semaine.

\subsection{Fabrications fromagères}

Les fromages ont été fabriqués selon le modèle Saint Paulin pour les pâtes pressées [8], et le modèle Sainte Maure pour les pâtes molles
[23], à partir de $11 \mathrm{~kg}$ de lait cru. Afin d'obtenir des temps de prise identiques, une quantité environ deux fois plus élevée de présure (CarlinTexel, $520 \mathrm{mg} \cdot \mathrm{L}^{-1}$ ) a été utilisée pour les laits à fraction écrémée de vache par rapport aux laits à fraction écrémée de chèvre. Six fromages d'environ 150-200 g ont été fabriqués par cuve. Après enrobage de cire, les PP ont été affinés 45 jours à $12{ }^{\circ} \mathrm{C}$, à $80-85 \%$ d'humidité relative (HR). L'affinage des $\mathrm{PM}$ a été réalisé à $12^{\circ} \mathrm{C}$, pendant 3 jours à $80 \%$ HR puis 10 jours à $90 \%$ HR.

\subsection{Analyses des fromages}

Les analyses ont été réalisées sur les fromages en début ( $24 \mathrm{~h}$ pour les PP, $48 \mathrm{~h}$ pour les PM) et en fin d'affinage ( $45 \mathrm{j}$ pour les $\mathrm{PP}, 13 \mathrm{j}$ pour les PM), sauf pour l'analyse des acides gras estérifiés réalisée seulement en début d'affinage et celle des taux de chlorures, calcium, composés volatils et caractéristiques organoleptiques effectuées uniquement en fin d'affinage. La croûte des fromages était préalablement retirée sur 2 à $3 \mathrm{~mm}$ d'épaisseur.

\subsubsection{Analyses physico-chimiques}

Le $\mathrm{pH}$ a été mesuré à l'aide d'une électrode de verre (Radiometer) sur le fromage râpé (PP) ou broyé (PM). La teneur en extrait sec a été analysée selon la norme FIL 4A [10] et exprimée en g pour $100 \mathrm{~g}$ de fromage. La teneur en matière grasse a été déterminée par la méthode acidobutyrométrique de Heiss [18] et exprimée en g pour $100 \mathrm{~g}$ de fromage. La teneur en azote total (NT) a été déterminée par la méthode Kjeldahl et utilisée pour calculer la teneur en protéines $(\mathrm{NT} \times 6,38)$. Extrait sec, teneur en matière grasse et teneur en protéines ont été utilisés pour calculer les rapports matière grasse sur extrait sec (G/S), protéines sur extrait sec (P/S) et humidité du fromage délipidé (H/FD) exprimés en pourcentage. Le taux de chlorures a été dosé à l'aide d'un chloruremètre (Corning 926) à électrode d'argent, après extraction selon la norme FIL $88 \mathrm{~A}$ [11] et exprimé en g pour $100 \mathrm{~g}$ d'eau. Le taux de calcium a été dosé par complexométrie selon la méthode de Pearce [28] et exprimé en $\mathrm{g}$ pour $100 \mathrm{~g}$ d'extrait sec.

\subsubsection{Protéolyse}

Les teneurs en azote soluble à pH 4,6 et en azote soluble dans l'acide phosphotungstique 
(NPT) ont été dosées selon la méthode de Gripon et al. [15]. Elles ont été exprimées en valeur relative par rapport à l'azote total et ont servi au calcul de la teneur en gros peptides dans l'azote total [(NS-NPT)/NT].

\subsubsection{Composition en acides gras}

Les acides gras ont été analysés par chromatographie en phase gazeuse après estérification. Ils ont été extraits selon la méthode de Deeth et al. [7] à partir de $1 \mathrm{~g}$ de fromage, après ajout d'étalons $\mathrm{C} 5: 0$ et C13:0. Les acides gras estérifiés ont été butylés selon la méthode de Decaen et Adda [6]. Les acides gras libres ont été séparés sur une résine anionique forte [27] puis méthylés selon la méthode FIL [12]. L'analyse chromatographique a été réalisée sur un chromatographe Carlo-Erba GC 8000 couplé à un intégrateur Spectra-Physics SP 4290 , équipé d'un injecteur on column, d'un détecteur FID chauffé à $260{ }^{\circ} \mathrm{C}$ et d'une colonne capillaire DBWAX ( $30 \mathrm{~m}$ long., $0,32 \mathrm{~mm}$ diam., $0,5 \mu \mathrm{m}$ phase). Le gaz vecteur (hélium) avait un débit de $2 \mathrm{~mL} \cdot \mathrm{min}^{-1}$ à $40^{\circ} \mathrm{C}$. $\mathrm{La}$ température du four s'élevait de 40 à $190^{\circ} \mathrm{C}$ à

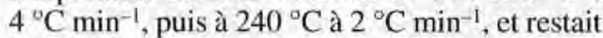
à $240^{\circ} \mathrm{C} 15 \mathrm{~min}$. La quantification des acides gras estérifiés a été réalisée par ajout de nonanoate de butyle lors de la butylation, celle des acides gras libres par l'ajout d'un décanoate de méthyle lors de la méthylation.

\subsubsection{Profil des composés volatils}

Les composés volatils ont été extraits, à partir de $100 \mathrm{~g}$ de fromage homogénéisés dans $600 \mathrm{~mL}$ d'eau ultra-pure, par hydrodistillation sous pression réduite, comme décrit par Dumont et Adda [9]. Les extraits aqueux obtenus ont été extraits par du dichlorométhane en conditions acides ( $\mathrm{pH}$ 2) ou neutres ( $\mathrm{pH} 7$ ), puis concentrés après ajout de pentanoate de pentyle comme étaIon interne [24]. Les composés volatils ont été analysés par chromatographie en phase gazeuse (chromatographe Hewlett-Packard HP 5890 series II) couplée à la spectrométrie de masse (spectromètre Nermag R 10-10 C quadrupole). Le chromatographe était équipé d'un injecteur split-splitless, d'une colonne capillaire DB-FFAP (30 $\mathrm{m}$ long., $0,32 \mathrm{~mm}$ diam., $0,25 \mu \mathrm{m}$ phase, J \& W Scientific). Le gaz vecteur (hélium) avait un débit de $2,8 \mathrm{~mL} \cdot \mathrm{min}^{-1}$ à $40^{\circ} \mathrm{C}$. La température du four s'élevait de 50 à $130^{\circ} \mathrm{C}$ à $6{ }^{\circ} \mathrm{C} \mathrm{min}$ min $^{-1}$, puis à $230^{\circ} \mathrm{C}$ à $12{ }^{\circ} \mathrm{C} \mathrm{min}{ }^{-1}$, l'injecteur était à $240{ }^{\circ} \mathrm{C}$, la ligne de transfert à $260^{\circ} \mathrm{C}$. L'enregistrement des spectres a été réalisé en impact électronique à $70 \mathrm{eV}$, avec un cycle de $0,8 \mathrm{~s}$, un balayage de 25 à $300 \mathrm{amu}$, et une température de source de $150^{\circ} \mathrm{C}$.

\subsubsection{Analyse sensorielle}

L'odeur, l'arôme, le goût et la texture des fromages affinés ont été analysés par un jury de 10 dégustateurs par la méthode des profils. Le jury a subi un entraînement préalable de 10 séances, au cours desquelles ont été choisis les descripteurs jugés les mieux adaptés pour la description des fromages, selon la méthodologie décrite dans la norme AFNOR NF V 09-021 [2]. Les descripteurs retenus ont été :

- « odeur » : intensité, chèvre, lait frais, lait acidifié, beurre, piquant ;

- « arôme » : intensité, chèvre, persistance chèvre, lait acidifié (PM uniquement), rance, piquant, persistance piquant ;

- « goût » : salé, acide, amer ;

- « texture » : collant, pâteux, granuleux, et pour les PM uniquement, sec, ferme, fondant.

Les descripteurs ont été notés sur une échelle non graduée de $10 \mathrm{~cm}$ (extrémité gauche : intensité nulle, extrémité droite : forte intensité). Les fromages ont été évalués en boxes individuels, éclairés avec une lumière rouge. Les quatre échantillons de fromage fabriqués le même jour ont été analysés au cours d'une même séance. Pour l'évaluation de l'odeur, chaque échantillon a été présenté dans un flacon brun de $30 \mathrm{~mL}$ rempli au tiers par du fromage râpé (PP) ou par des cubes de 0,5 à $1 \mathrm{~cm}$ de côté (PM). Pour l'évaluation de l'arôme, du goût et de la texture, chaque échantillon a été présenté en boîte de Pétri. Après élimination de la croûte, les PP ont été présentés sous forme de cubes d'environ $1,2 \mathrm{~cm}$ de côté, et les PM sous forme de tranches de $1,5 \mathrm{~cm}$ de hauteur coupées transversalement. Les échantillons ont été dégustés sans retour en arrière dans un ordre préétabli suivant un plan expérimental en carré latin modifié [25].

\subsection{Analyses statistiques}

Les traitements statistiques ont été faits séparément pour chaque type de fromage PP et PM, avec le logiciel STAT-ITCF (1991, Institut technique des céréales et fourrages, Paris). Les moyennes et écarts types ont été calculés pour chaque variable sur les quatre fabrications. Des analyses de variance à deux facteurs (origine du lait écrémé $x$ origine de la crème) en blocs (jour 
de fabrication) ont été effectuées sur les variables physico-chimiques et biochimiques. Des analyses de variance à trois facteurs (type de lait écrémé $\times$ type de crème $\times$ dégustateur) en blocs (jour de fabrication) ont été effectuées sur les variables sensorielles. Des comparaisons de moyennes des quatre types de fromages ont été effectuées selon la méthode de Newmann-Keuls [5]. Une analyse en composantes principales a été réalisée sur les composés volatils influencés de façon significative par les fractions du lait en analyse de variance.

\section{RÉSULTATS}

\subsection{Composition physico-chimique et protéolyse}

La fraction grasse semble avoir peu d'effet sur la composition physico-chimique des fromages, sauf sur l'ES et l'H/FD des $\mathrm{PM}$ en fin d'affinage (tableaux I et II). Au contraire, la fraction écrémée intervient sur

Tableau I. Composition physico-chimique des fromages à pâte pressée. Moyennes des quatre fabrications pour chaque type de fromage.

Table I. Physico-chemical composition of semi-hard cheeses. Means of four replicates for each type of cheese.

\begin{tabular}{|c|c|c|c|c|c|c|c|}
\hline & VV & $\mathrm{VC}$ & $\mathrm{CV}$ & $\mathrm{CC}$ & LE & MG & $\mathrm{LE} \times \mathrm{MG}$ \\
\hline \multicolumn{8}{|l|}{ Fromages au démoulage } \\
\hline $\mathrm{pH}$ & $5,10^{\mathrm{a}, \mathrm{b}}$ & $5,06^{\mathrm{b}}$ & $5,20^{\mathrm{a}}$ & $5,19^{\mathrm{a}}$ & $* *$ & NS & NS \\
\hline $\mathrm{ES}(\%)$ & 44,8 & 46,7 & 46,9 & 47,7 & $*$ & * & NS \\
\hline $\mathrm{H} / \mathrm{FD}(\%)$ & $70,0^{\mathrm{a}}$ & $68,7^{\mathrm{a}, \mathrm{b}}$ & $67,6^{\mathrm{b}, \mathrm{c}}$ & $66,9^{c}$ & $* *$ & * & * \\
\hline $\mathrm{G} / \mathrm{S}(\%)$ & $47,0^{\mathrm{a}, \mathrm{b}}$ & $48,0^{\mathrm{a}}$ & $45,8^{\mathrm{b}}$ & $45,8^{b}$ & $* *$ & NS & NS \\
\hline $\mathrm{P} / \mathrm{S}(\%)$ & 45,8 & 45,6 & 46,6 & 46,7 & $*$ & NS & NS \\
\hline Gros peptides/NT (\%) & $8,8^{\mathrm{a}}$ & $8,6^{\mathrm{a}}$ & $6,8^{b}$ & $6,8^{b}$ & **** & NS & NS \\
\hline NPT/NT $(\%)$ & 0,53 & 0,55 & 0,42 & 0,63 & NS & NS & NS \\
\hline AGL totaux (mmol/100 g MG) & $2,05^{\mathrm{a}}$ & $1,43^{b}$ & $1,49^{b}$ & $0,73^{\mathrm{c}}$ & $* * *$ & $* * *$ & NS \\
\hline \multicolumn{8}{|l|}{ Fromages en fin d'affinage } \\
\hline $\mathrm{pH}$ & $5,11^{\mathrm{b}}$ & $5,12^{b}$ & $5,44^{\mathrm{a}}$ & $5,43^{\mathrm{a}}$ & $* * *$ & NS & NS \\
\hline $\mathrm{ES}(\%)$ & $47,7^{\mathrm{b}}$ & $49,3^{\mathrm{a}, \mathrm{b}}$ & $50,1^{\mathrm{a}}$ & $49,3^{\mathrm{a}, \mathrm{b}}$ & * & NS & $*$ \\
\hline $\mathrm{H} / \mathrm{FD}(\%)$ & $66,5^{\mathrm{a}}$ & $65,8^{\mathrm{a}, \mathrm{b}}$ & $64,5 b$ & $64,9^{a, b}$ & $* *$ & NS & NS \\
\hline $\mathrm{Cl} / \mathrm{H}_{2} \mathrm{O}(\%)$ & 3,4 & 3,4 & 3,2 & 3,3 & NS & NS & NS \\
\hline $\mathrm{Ca} / \mathrm{ES}(\%)$ & $1,25^{\mathrm{b}}$ & $1,23 b$ & $1,52^{\mathrm{a}}$ & $1,56^{\mathrm{a}}$ & $* * *$ & NS & NS \\
\hline $\mathrm{G} / \mathrm{S}(\%)$ & $44,9^{b}$ & $46,4 \mathrm{a}$ & $45,3^{\mathrm{a}, \mathrm{b}}$ & $44,3^{b}$ & NS & NS & $* *$ \\
\hline $\mathrm{P} / \mathrm{S}(\%)$ & $44,3 \mathrm{a}$ & $43,1^{\mathrm{b}}$ & $44,8^{\mathrm{a}}$ & $45,4^{\mathrm{a}}$ & $* *$ & NS & * \\
\hline Gros peptides/NT (\%) & 10,5 & 11,0 & 10,8 & 11,2 & NS & NS & NS \\
\hline NPT/NT $(\%)$ & $2,67^{\mathrm{b}}$ & $3,07^{\mathrm{b}}$ & $4,32^{\mathrm{a}}$ & $4,06^{\mathrm{a}}$ & $* *$ & NS & NS \\
\hline AGL totaux (mmol/100 $\mathrm{g} \mathrm{MG})$ & $2,78^{\mathrm{a}}$ & $2,26^{b, c}$ & $2,54^{\mathrm{a}, \mathrm{b}}$ & $1,77^{\mathrm{d}}$ & $* *$ & $* * *$ & NS \\
\hline
\end{tabular}

ES : extrait sec, H/FD : humidité du fromage dégraissé, G/S : gras / sec, P/S : protéines / extrait sec, NT : azote total, NPT : azote soluble dans l'acide phosphotungstique, AGL : acides gras libres. VV, VC, CV, CC : la première lettre désigne la nature de la fraction écrémée, la deuxième lettre désigne la nature de la fraction grasse (V : vache, $\mathrm{C}$ : chèvre). Résultat de l'analyse de variance : LE : effet du lait écrémé, MG : effet de la matière grasse, LE $\times$ MG : interaction entre les deux facteurs. $* * *: P<0,001 ; * *: P<0,01 ; *: P<0,05$; NS : non significatif. Comparaison de moyennes : les fromages sont classés en différents groupes $\left({ }^{a}, b, c, d\right)$ par le test de Newmann-Keuls.

ES: dry matter, H/FD: water in non-fat cheese, G/S: fat / dry matter, P/S: proteins / dry matter, NT: total nitrogen, NPT: soluble nitrogen in phosphotungstic acid, AGL : free fatty acids. VV, VC, CV, CC: the first letter refers to the nature of the skimmed milk fraction, the second letter refers to the nature of the fatty fraction (V: cow, C: goat). Result of variance analysis: LE : skimmed milk fraction effect, MG: fatty fraction effect, LE $\times$ MG: interaction between the two factors. $* * *: P<0.001 ; * *: P<0.01 ; *: P<0.05$; NS: non significant. Comparison of means: the cheeses are classified in different groups $(a, b, c, d)$ by the test of Newmann-Keuls. 
Tableau II. Composition physico-chimique des fromages à pâte molle. Moyennes et écarts types des quatre fabrications pour chaque type de fromage.

Table II. Physico-chemical composition of soft cheeses. Means of four replicates for each type of cheese.

\begin{tabular}{|c|c|c|c|c|c|c|c|}
\hline & VV & VC & $\mathrm{CV}$ & $\mathrm{CC}$ & LE & MG & $\mathrm{LE} \times \mathrm{MG}$ \\
\hline \multicolumn{8}{|l|}{ Fromages au démoulage } \\
\hline $\mathrm{pH}$ & $4,28^{\mathrm{a}}$ & $4,27^{a}$ & $4,25^{\mathrm{b}}$ & $4,25^{b}$ & $* * *$ & * & NS \\
\hline ES (\%) & 37,0 & 36,7 & 36,0 & 37,2 & NS & NS & NS \\
\hline $\mathrm{H} / \mathrm{FD}(\%)$ & 78,0 & 78,3 & 79,4 & 78,1 & NS & NS & NS \\
\hline $\mathrm{G} / \mathrm{S}(\%)$ & 52,0 & 52,1 & 53,9 & 52,7 & * & NS & NS \\
\hline $\mathrm{P} / \mathrm{S}(\%)$ & $38,0^{\mathrm{a}}$ & $38,2^{a}$ & $36,9^{b}$ & $37,3^{b}$ & $* * *$ & NS & NS \\
\hline Gros peptides/NT (\%) & $14,4^{\mathrm{a}}$ & $14,2^{\mathrm{a}}$ & $12,6^{\mathrm{b}}$ & $12,0^{b}$ & $* * *$ & NS & NS \\
\hline NPT/NT (\%) & 1,94 & 2,06 & 1,91 & 2,00 & NS & NS & NS \\
\hline AGL totaux (mmol/100 g MG) & $2,22^{\mathrm{a}}$ & $1,30^{c}$ & $1,66^{\mathrm{b}}$ & $0,74^{d}$ & $* * *$ & $* * *$ & \\
\hline \multicolumn{8}{|l|}{ Fromages en fin d'affinage } \\
\hline $\mathrm{pH}$ & 4,82 & 4,75 & 4,77 & 4,79 & NS & NS & NS \\
\hline $\mathrm{ES}(\%)$ & $45,4^{\mathrm{b}}$ & $48,0^{\mathrm{a}}$ & $45,4^{b}$ & $49,2^{\mathrm{a}}$ & NS & $* * *$ & NS \\
\hline $\mathrm{H} / \mathrm{FD}(\%)$ & $72,4^{\mathrm{a}}$ & $70,3^{b}$ & $72,4^{\mathrm{a}}$ & $69,5^{\mathrm{b}}$ & NS & $* * *$ & NS \\
\hline $\mathrm{Cl} / \mathrm{H}_{2} \mathrm{O}(\%)$ & 3,0 & 3,1 & 3,5 & 3,5 & $*$ & NS & NS \\
\hline $\mathrm{Ca} / \mathrm{ES}^{2}(\%)$ & 0,13 & 0,17 & 0,10 & 0,17 & NS & NS & NS \\
\hline G/S $(\%)$ & 54,0 & 54,2 & 54,2 & 54,5 & NS & NS & NS \\
\hline $\mathrm{P} / \mathrm{S}(\%)$ & 39,3 & 39,7 & 39,5 & 39,7 & NS & NS & NS \\
\hline Gros peptides/NT (\%) & 24,0 & 21,6 & 22,7 & 22,1 & NS & NS & NS \\
\hline NPT/NT (\%) & 5,64 & 4,81 & 5,64 & 5,60 & NS & NS & NS \\
\hline AGL totaux (mmol/100 g MG) & $2,58^{\mathrm{a}}$ & $1,43^{\mathrm{c}}$ & $2,18^{\mathrm{b}}$ & $1,03^{\mathrm{d}}$ & $* *$ & $* * *$ & NS \\
\hline
\end{tabular}

Abréviations : voir tableau I.

Abbreviations: see table I.

la plupart des critères étudiés dans les PP (pH, ES, H/FD, G/S, P/S en début d'affinage, $\mathrm{pH}, \mathrm{ES}, \mathrm{H} / \mathrm{FD}, \mathrm{Ca}, \mathrm{P} / \mathrm{S}$ en fin d'affinage). Dans les PM, cette fraction intervient principalement en début d'affinage $(\mathrm{pH}$, $\mathrm{G} / \mathrm{S}, \mathrm{P} / \mathrm{S})$.

En ce qui concerne la protéolyse, les différences entre fromages semblent limitées. Au démoulage, la fraction écrémée de chèvre donne, pour les fromages des deux technologies, une proportion de gros peptides moins élevée que la fraction écrémée de vache. En revanche, on note une tendance inverse pour la protéolyse secondaire en fin d'affinage, puisque les PP à lait écrémé de chèvre ont des teneurs en NPT/NT plus fortes que les fromages à lait écrémé de vache. Dans les PM, les indicateurs de pro- téolyse sont deux fois plus élevés que dans les PP, mais aucune différence de protéolyse n'est observée entre fromages.

\subsection{Teneurs en AGL et compositions en acides gras}

Les teneurs en AGL totaux sont similaires entre PP et PM, et dépendent fortement des fractions écrémées et grasses (tableaux I et II). Elles sont plus faibles en présence d'une fraction de lait de chèvre (écrémée ou grasse), du début à la fin de l'affinage. En ce qui concerne les taux d'AGL pris individuellement, presque tous dépendent de l'origine de la fraction grasse, mais seuls les C6:0 (PP uniquement), C8:0 et C10:0 sont en 
quantités plus fortes en présence de matière grasse de chèvre (tableaux III et $I V$ ). La fraction écrémée de chèvre donne des teneurs plus faibles en AGL, pour le C14:0 et les acides gras à nombre supérieur d'atomes de carbone dans les PP, pour tous les acides gras, sauf le C6:0 dans les PM.

La composition en AGL (résultats non présentés) est très proche de la composition en acides gras des triglycérides en début comme en fin d'affinage, et varie peu au cours de l'affinage, malgré une légère augmentation des acides gras courts ( $\mathrm{C} 4: 0$ et C6:0) et une légère diminution du C18:0 (et C18:1 pour les PP). Les triglycérides de chèvre contiennent des proportions plus fortes en C6:0, C8:0, C10:0, C12:0 que les triglycérides de vache, et plus faibles pour les autres acides gras (figure l).

\subsection{Composés volatils}

Les composés volatils ont été dosés sur deux séries de chaque type de fromage. Dans les PP, 43 composés ont été quantifiés (13 acides gras, dont 4 ramifiés, 11 cétones, 5 alcools, 5 composés aromatiques, 4 esters, 4 lactones, 1 composé soufré), et, dans les PM, 47 composés ( 2 acides gras ramifiés, 1 ester et 1 inconnu en plus). Les PM ont généralement une teneur moyenne en composés volatils de 2 à 10 fois supérieure aux PP. D'une façon générale, les quantités trouvées étant très variables entre fromages et entre jours de fabrication, il est difficile de mettre en évidence un effet de l'une ou l'autre fraction sur les composés volatils pris individuellement. Cependant, l'analyse en composantes principales montre qu'il est

Tableau III. Quantités d'acides gras libres des fromages à pâte pressée en fin d'affinage. Moyennes des quatre fabrications pour chaque type de fromage en $\mu$ moles / $100 \mathrm{~g} \mathrm{MG}$

Table III. Amounts of free fatty acids of semi-hard cheeses at the end of ripening. Means of four replicates for each type of cheese in $\mu$ moles / $100 \mathrm{~g}$ fat matter.

\begin{tabular}{|c|c|c|c|c|c|c|c|}
\hline & VV & VC & $\mathrm{CV}$ & $\mathrm{CC}$ & LE & MG & $\mathrm{LE} \times \mathrm{MG}$ \\
\hline $\mathrm{C} 4: 0$ & $263,1^{\mathrm{a}}$ & $143,4^{b}$ & $241,7^{\mathrm{a}}$ & $155,0^{c}$ & NS & $* * *$ & NS \\
\hline C6:0 & 84,6 & 86,0 & 71,0 & 85,6 & NS & $* *$ & NS \\
\hline C8:0 & $42,8^{\mathrm{b}}$ & $72,8^{\mathrm{a}}$ & $43,4^{b}$ & $75,7^{\text {a }}$ & NS & $* * *$ & NS \\
\hline $\mathrm{C} 10: 0$ & $90,6^{b}$ & $219,5^{\mathrm{a}}$ & $106,3^{\mathrm{b}}$ & $261,0^{\mathrm{a}}$ & NS & $*$ & NS \\
\hline $\mathrm{C} 10: 1$ & 10,2 & 4,8 & 9,5 & 5,4 & NS & NS & NS \\
\hline C12:0 & 122,5 & 122,3 & 118,7 & 130,4 & NS & NS & NS \\
\hline C12:1 & 3,0 & 1,5 & 3,0 & 1,2 & nd & nd & nd \\
\hline C13:0 & 4,3 & 3,1 & 4,3 & 5,0 & nd & nd & nd \\
\hline $\mathrm{C} 14: 0$ & $344,4^{a}$ & $215,5^{\mathrm{c}}$ & $299,4^{\mathrm{b}}$ & $174,2^{\mathrm{d}}$ & $* *$ & $* * *$ & NS \\
\hline $\mathrm{C} 14: 1$ & 25,4 & 7,6 & 20,8 & 2,1 & nd & nd & nd \\
\hline C15:0 & $65,7^{\mathrm{a}}$ & $32,6^{c}$ & $52,5^{b}$ & $21,4^{d}$ & $* *$ & $* * *$ & NS \\
\hline C16:0 & $749,4^{a}$ & $531,6^{b}$ & $673,3^{a}$ & $408,5^{c}$ & $* *$ & $* * *$ & NS \\
\hline C16:1 & $41,9^{a}$ & $21,0^{\mathrm{b}}$ & $43,6^{a}$ & $13,8^{\mathrm{c}}$ & NS & $* * *$ & * \\
\hline C17:0 & $15,7^{\mathrm{a}}$ & $11,2^{\mathrm{b}}$ & $14,3^{a}$ & $7,8^{c}$ & $* *$ & $* * *$ & NS \\
\hline C18:0 & $206,5^{\mathrm{a}}$ & $190,5^{a}$ & $184,5^{\mathrm{a}}$ & $119,7^{b}$ & $* *$ & $* *$ & NS \\
\hline C18:1 & $566,0^{\mathrm{a}}$ & $471,9^{\mathrm{a}}$ & $525,0^{\mathrm{a}}$ & $250,0^{b}$ & $* * *$ & $* * *$ & $* *$ \\
\hline C18:2 & $102,8^{\mathrm{a}}$ & $98,7^{\mathrm{a}}$ & $87,5^{\mathrm{a}}$ & $40,0^{\mathrm{b}}$ & $* * *$ & $* *$ & $* *$ \\
\hline C18:3 & $28,1^{\mathrm{a}}$ & $20,0^{c}$ & $25,5^{\mathrm{b}}$ & $10,0^{\mathrm{d}}$ & $* *$ & $* * *$ & NS \\
\hline C20:0 & 3,5 & 2,9 & 4,6 & 1,3 & nd & nd & nd \\
\hline C20:1 & 7,5 & 3,1 & 6,2 & 0,6 & nd & nd & nd \\
\hline
\end{tabular}

Abréviations : voir tableau I. nd : non déterminé.

Abbreviations: see table I. nd: undetermined. 
Tableau IV. Quantités d'acides gras libres des fromages à pâte molle en fin d'affinage. Moyennes des quatre fabrications pour chaque type de fromage en $\mu \mathrm{mol} / 100 \mathrm{~g} \mathrm{MG}$.

Table IV. Amounts of free fatty acids of soft cheeses at the end of ripening. Means of four replicates for each type of cheese in $\mu \mathrm{mol} / 100 \mathrm{~g}$ fat matter.

\begin{tabular}{|c|c|c|c|c|c|c|c|}
\hline & VV & $\mathrm{VC}$ & $\mathrm{CV}$ & $\mathrm{CC}$ & $\mathrm{LE}$ & MG & $\mathrm{LE} \times \mathrm{MG}$ \\
\hline $\mathrm{C} 4: 0$ & $91,2^{\mathrm{b}}$ & $43,9^{b}$ & $167,6^{\mathrm{a}}$ & $96,3^{\mathrm{b}}$ & $* *$ & $* *$ & NS \\
\hline C6:0 & 53,0 & 44,1 & 69,1 & 68,5 & * & NS & NS \\
\hline C8:0 & $71,8^{b}$ & $80,6^{\mathrm{a}}$ & $44,1^{d}$ & $52,4^{c}$ & $* * *$ & $* *$ & NS \\
\hline $\mathrm{C} 10: 0$ & $126,8^{\mathrm{c}}$ & $180,2^{\mathrm{a}}$ & $102,2^{\mathrm{d}}$ & $148,5^{b}$ & $* * *$ & $* * *$ & NS \\
\hline C10:1 & 11,5 & 11,1 & 7,4 & 3,5 & * & NS & NS \\
\hline $\mathrm{C} 12: 0$ & $127,2^{\mathrm{a}}$ & $99,7^{\mathrm{b}}$ & $95,7^{\mathrm{b}}$ & $69,6^{\mathrm{c}}$ & $* * *$ & $* * *$ & NS \\
\hline $\mathrm{C} 12: 1$ & 3,2 & 1,8 & 2,2 & 0,7 & nd & nd & nd \\
\hline C13:0 & 5,2 & 2,9 & 3,7 & 1,7 & nd & nd & nd \\
\hline $\mathrm{Cl} 4: 0$ & $310,3^{\mathrm{a}}$ & $163,7^{\mathrm{c}}$ & $235,9^{b}$ & $90,1^{d}$ & $* * *$ & $* * *$ & NS \\
\hline C14:1 & 24,3 & 6,4 & 17,1 & 1,0 & nd & nd & nd \\
\hline C15:0 & $58,7^{a}$ & $25,6^{\mathrm{c}}$ & $45,2^{\mathrm{b}}$ & $13,9^{d}$ & $* *$ & $* * *$ & NS \\
\hline $\mathrm{C} 16: 0$ & $663,5^{\mathrm{a}}$ & $357,7^{\mathrm{c}}$ & $529,9^{b}$ & $222,3^{\mathrm{d}}$ & $* * *$ & $* * *$ & NS \\
\hline C16:1 & $53,1^{\mathrm{a}}$ & $18,8^{\mathrm{c}}$ & $42,6^{b}$ & $10,2^{\mathrm{d}}$ & $* *$ & $* * *$ & NS \\
\hline $\mathrm{C} 17: 0$ & $14,8^{\mathrm{a}}$ & $6,8^{c}$ & $12,3^{b}$ & $4,0^{\mathrm{d}}$ & $* * *$ & $* * *$ & NS \\
\hline C18:0 & $195,6^{\mathrm{a}}$ & $103,6^{\mathrm{b}}$ & $163,2^{\mathrm{a}}$ & $67,5^{b}$ & $* * *$ & $* * *$ & NS \\
\hline C18:1 & $606,1^{\mathrm{a}}$ & $219,1^{\mathrm{c}}$ & $518,2^{b}$ & $134,5^{\mathrm{d}}$ & * & $* * *$ & NS \\
\hline $\mathrm{C} 18: 2$ & $114,4^{\mathrm{a}}$ & $45,6^{c}$ & $87,8^{b}$ & $25,8^{\mathrm{d}}$ & $* *$ & $* * *$ & NS \\
\hline C18:3 & $36,4^{\mathrm{a}}$ & $16,2^{c}$ & $28,1^{\mathrm{b}}$ & $10,8^{d}$ & $* *$ & $* * *$ & NS \\
\hline $\mathrm{C} 20: 0$ & 3,3 & 1,7 & 2,4 & 0,9 & nd & nd & nd \\
\hline $\mathrm{C} 20: 1$ & 8,3 & 2,1 & 5,9 & 10,7 & nd & nd & nd \\
\hline
\end{tabular}

Abréviations : voir tableau I. nd : non déterminé.

Abbreviations: see table I. nd: undetermined.

possible de discriminer les fromages en fonction de l'origine des fractions écrémée et grasse à partir de quelques-uns de ces composés.

Pour les PP, l'axe 1 ( $57 \%$ de la variance totale) discrimine les fromages selon la nature de la fraction écrémée, alors que sur l'axe $2(16 \%)$, les fromages sont séparés 2 à 2 par la nature de leur fraction grasse (figure $2 b$ ). Sur l'axe 1, la fraction écrémée de chèvre a tendance à donner des fromages plus riches en 2-méthyl 1-butanol, 3-hydroxy 2-butanone et 1-penténol, et moins riches en acides gras minoritaires (C5:0, C7:0, C9:0, 4MeC6:0), méthyl-cétones (2-octanone, 2-nonanone), alcools (2-heptanol, 7-octèn 2-ol) (figure 2a). Sur l'axe 2, la frac- tion grasse de chèvre a tendance à donner des teneurs en acides gras minoritaires (C7:0, 4MeC6:0, 4MeC8:0) plus importantes que celle de vache.

Pour les PM, le plan 1-2 représente $66 \%$ de la variance totale. Les fromages sont discriminés en fonction de leur fraction écrémée et de leur fraction grasse par deux axes indépendants situés entre les axes 1 et 2 (figure $3 b$ ). Alors que les alcools, les méthylcétones et les esters se répartissent entre les quatre groupes de fromages, les acides gras minoritaires (C9:0, 4MeC6:0, 4MeC8:0, 4EtC8:0) sont plus abondants dans les fromages pur chèvre et les lactones dans les fromages pur vache (figure $3 a$ ). 


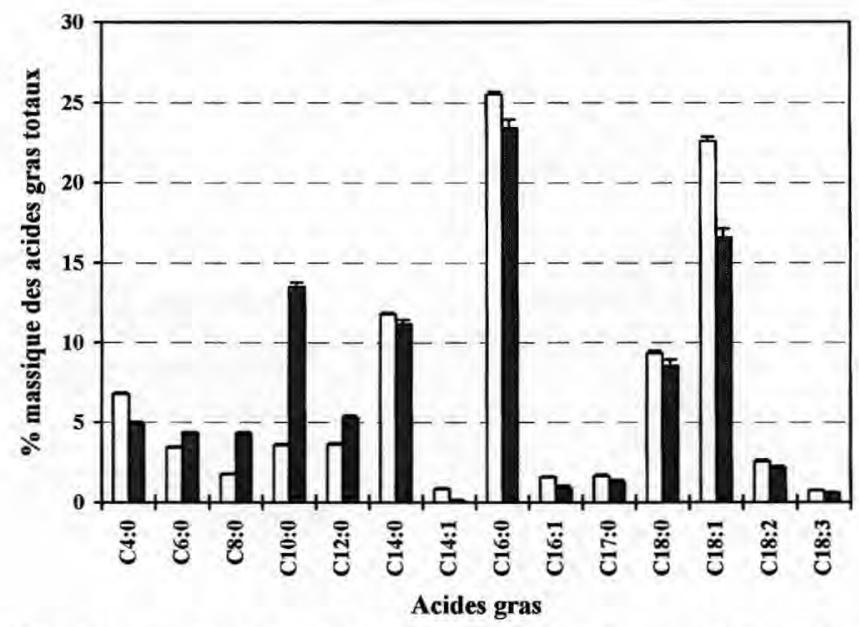

Figure 1. Profil des principaux acides gras des triglycérides des fromages en fonction de l'origine de la matière grasse : moyennes et écarts types des résultats obtenus sur les pâtes pressées et sur les pâtes molles. $\square$ : matière grasse de vache, $\mathbf{\square}$ : matière grasse de chèvre.

Figure 1. Profile of the main fatty acids of the triglycerides of the cheeses according to the origin of the fatty fraction : means and standard deviations of the results obtained on the semi-hard and soft cheeses. $\square$ : cow's fatty fraction, $\mathbf{Q}$ : goat's fatty fraction.

\subsection{Analyse sensorielle}

\subsubsection{Flaveur (odeur, arôme, goût)}

Pour les PP, il semble que c'est la fraction grasse dont l'influence est la plus grande sur la flaveur (tableau $V$ ). On note en particulier que la matière grasse de chèvre donne des notes plus fortes pour les descripteurs « chèvre » et « intensité globale », aussi bien pour l'odeur que pour l'arôme, mais moins fortes pour les odeurs « lait frais » et « beurre », et pour l'amertume. Concernant la fraction écrémée, le lait de chèvre a un léger effet positif sur l'arôme "chèvre ", mais négatif sur les arômes « rance » et «piquant» et sur la saveur acide.

Pour les PM, la fraction grasse du lait de chèvre a une forte influence positive sur l'odeur et l'arôme « chèvre ", et un effet négatif sur les odeurs « lait frais » et « beurre » et sur l'arôme « rance » (tableau VI). La fraction écrémée de chèvre a également une influence positive sur l'odeur « chèvre » et dans une moindre mesure sur l'arôme
« chèvre », et favoriserait l'arôme " piquant » et la saveur acide. La fraction écrémée de vache semble favoriser essentiellement l'amertume.

\subsubsection{Texture}

Pour les PP, les trois critères analysés semblent uniquement influencés par l'origine de la fraction écrémée (tableau $V$ ), les fromages à fraction écrémée de chèvre étant plus granuleux, mais moins collants et pâteux. Pour les PM, les caractères pâteux et sec semblent également influencés par la fraction écrémée, avec des notes plus faibles pour le lait de chèvre. À l'inverse des PP, la matière grasse interviendrait également, celle de chèvre donnant des fromages plus fermes et fondants (tableau VI).

\section{DISCUSSION ET CONCLUSIONS}

La flore de surface (essentiellement Penicillium) des PM est connue pour avoir 
a)

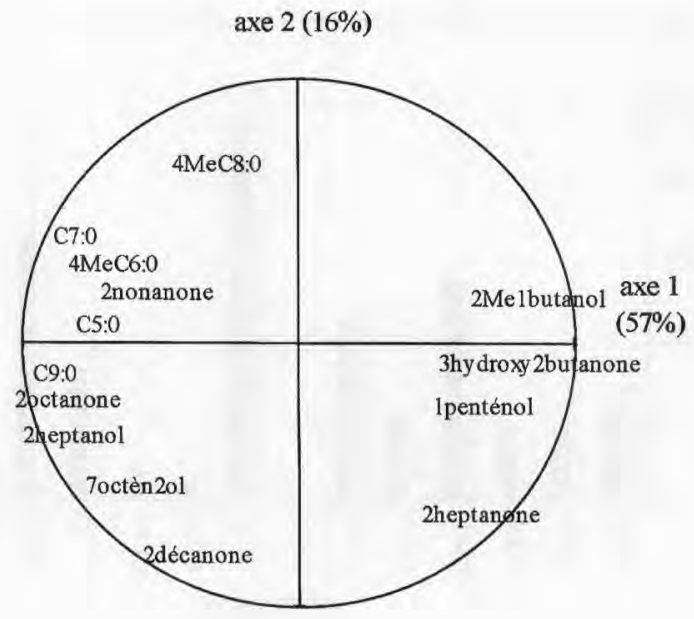

b)

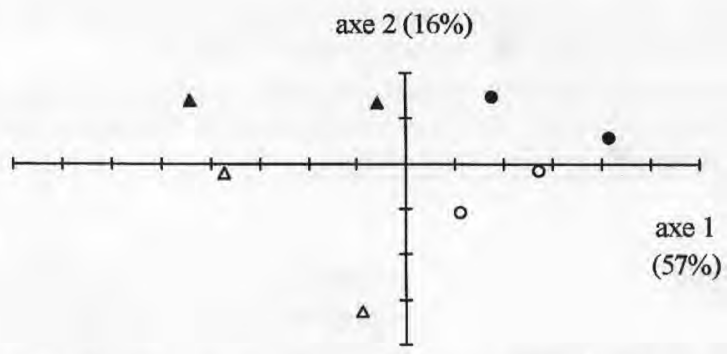

Figure 2. Plan 1-2 de l'analyse en composantes principales réalisée avec les composés volatils des fromages à pâte pressée en fin d'affinage (deux répétitions). a : cercle des corrélations ; $\mathbf{b}$ : représentation des échantillons. $\triangle: \mathrm{VV}, \boldsymbol{\Lambda}: \mathrm{VC}, \mathrm{O}: \mathrm{CV}, \boldsymbol{O}: \mathrm{CC}$.

Figure 2. 1-2 map of principal components analysis realised with volatile components of the semihard cheeses at the end of ripening (two replicates). a: circle of correlations; b: representation of samples. $\triangle: \mathrm{VV}, \boldsymbol{\Delta}: \mathrm{VC}, \mathrm{O}: \mathrm{CV}, \mathbf{O}: \mathrm{CC}$.

d'importantes activités protéolytique, lipolytique et oxydative $[14,22]$. Cela pourrait expliquer en partie que le niveau de protéolyse et la concentration en composés volatils soient plus élevés dans les PM que dans les PP. En ce qui concerne la lipolyse, la courte durée d'affinage des PM (13 jours) peut expliquer qu'elle soit faible dans ces fromages (moins de $1 \%$ ) et équivalente à celle des PP, car l'activité lipolytique de Penicillium camembertii ne se développe que tardivement dans les fromages à croûte fleurie (au-delà du dixième jour) $[14,22]$.

\subsection{Protéolyse}

La différence de protéolyse observée au démoulage entre fromages faits avec des laits écrémés différents porte sur la protéolyse primaire. Cette différence est sans doute due à la dose de présure (chymosine), deux fois plus faible dans les fromages faits à partir de lait écrémé de chèvre. De plus, la caséine de chèvre serait moins sensible à la chymosine que la caséine de vache [32]. Or, cette enzyme est, avec la plasmine, le principal agent de la protéolyse primaire dans les fromages [13]. Les différences de pro- 
a)

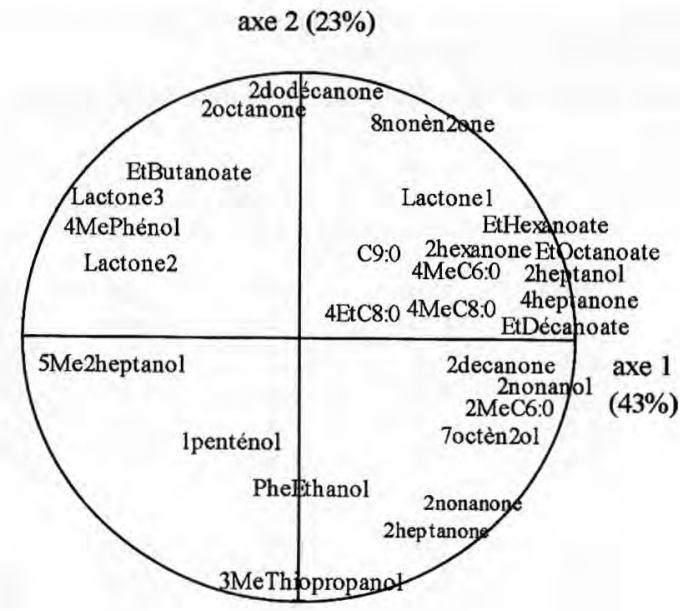

axe $2(23 \%)$

b)

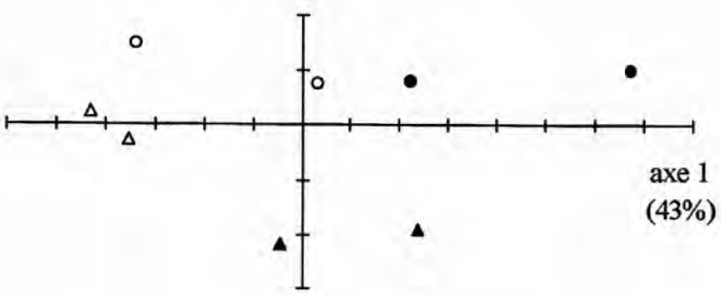

Figure 3. Plan 1-2 de l'analyse en composantes principales réalisée avec les composés volatils des fromages à pâte molle en fin d'affinage (deux répétitions). a : cercle des corrélations ; b : représentation des échantillons. $\triangle: \mathrm{VV}, \boldsymbol{\Delta}: \mathrm{VC}, \mathrm{O}: \mathrm{CV}, \bullet: \mathrm{CC}$.

Figure 3. 1-2 map of principal components analysis realised with volatile components of the soft cheeses at the end of ripening (two replicates). a: circle of correlations; b: representation of samples. $\triangle: \mathrm{VV}, \boldsymbol{\Delta}: \mathrm{VC}, \mathrm{O}: \mathrm{CV}, \boldsymbol{\theta}: \mathrm{CC}$.

téolyse secondaire, observées dans les PP en fin d'affinage, sont vraisemblablement liées au $\mathrm{pH}$, plus élevé dans les fromages à lait écrémé de chèvre.

\subsection{Acides gras libres et composés volatils}

La présence de lait de chèvre, que ce soit la fraction écrémée ou la fraction grasse, a comme conséquence, quelle que soit la technologie, un taux d'AGL plus faible dès le début de l'affinage. Bien qu'aucune mesure d'indice de lipolyse n'ait été réalisée sur le lait, on peut penser que les conditions de collecte et de transport du lait de vache, beaucoup moins favorables que celles du lait de chèvre, ont entraîné une lipolyse significative. L'influence de l'origine de la fraction écrémée sur la teneur en AGL peut s'expliquer en partie par la présence d'AGL dans la phase aqueuse. Une partie de ces composés, notamment les plus courts, plus hydrophiles, est susceptible de se solubiliser dans la fraction écrémée. La similitude de composition en acides gras entre triglycérides et AGL indique que l'activité lipoly- 
Tableau V. Caractéristiques sensorielles des fromages à pâte pressée en fin d'affinage. Moyennes des quatre fabrications pour chaque type de fromage.

Table V. Sensory characteristics of semi-hard cheeses at the end of ripening. Means of four replicates for each type of cheese.

\begin{tabular}{|c|c|c|c|c|c|c|c|}
\hline VV & VC & $\mathrm{CV}$ & $\mathrm{CC}$ & LE & & MG & $\mathrm{LE} \times \mathrm{MG}$ \\
\hline \multicolumn{8}{|l|}{ Odeur } \\
\hline Chèvre & $2,0^{a, b}$ & $3,0^{\mathrm{a}}$ & $1,8^{\mathrm{b}}$ & $3,0^{\mathrm{a}}$ & NS & $* * *$ & NS \\
\hline Lait frais & $1,4^{\mathrm{a}}$ & $1,2^{\mathrm{a}, \mathrm{b}}$ & $1,3^{\mathrm{a}, \mathrm{b}}$ & $0,9^{b}$ & NS & * & NS \\
\hline Lait acidifié & 1,3 & 1,4 & 1,2 & 1,4 & NS & NS & NS \\
\hline Beurre & $2,0^{\mathrm{a}}$ & $1,5^{\mathrm{a}, \mathrm{b}}$ & $1,8^{\mathrm{a}, \mathrm{b}}$ & $1,4^{\mathrm{b}}$ & NS & $* *$ & NS \\
\hline Piquant & 1,5 & 1,7 & 1,4 & 1,3 & NS & NS & NS \\
\hline Intensité & 3,8 & 4,2 & 3,5 & 3,9 & NS & $*$ & NS \\
\hline \multicolumn{8}{|l|}{ Arôme } \\
\hline Chèvre & $0,5^{\mathrm{c}}$ & $3,0^{\mathrm{b}}$ & $0,9^{c}$ & $3,8^{\mathrm{a}}$ & * & $* * *$ & NS \\
\hline Persistance chèvre & $0,4^{\mathrm{b}}$ & $2,2 a$ & $0,4^{\mathrm{b}}$ & $2,3^{\mathrm{a}}$ & NS & $* * *$ & NS \\
\hline Piquant & 2,8 & 2,8 & 2,8 & 1,8 & NS & NS & NS \\
\hline Persistance piquant & 2,1 & 2,0 & 1,5 & 1,3 & $* *$ & NS & NS \\
\hline Rance & $1,4^{\mathrm{a}}$ & $1,1^{\mathrm{a}, \mathrm{b}}$ & $0,8^{\mathrm{b}}$ & $0,7^{\mathrm{b}}$ & $* * *$ & NS & NS \\
\hline Intensité & $3,8^{b}$ & $4,5 \mathrm{a}$ & $3,4^{b}$ & $4,3^{\mathrm{a}}$ & NS & $* * *$ & NS \\
\hline \multicolumn{8}{|l|}{ Goût } \\
\hline Salé & $4,6^{\mathrm{a}}$ & $4,8^{\mathrm{a}}$ & $4,7^{\mathrm{a}}$ & $4,2^{\mathrm{b}}$ & NS & NS & $*$ \\
\hline Acide & $3,9^{\mathrm{a}}$ & $4,0^{\mathrm{a}}$ & $2,6^{\mathrm{b}}$ & $2,1^{\mathrm{b}}$ & $* * *$ & NS & NS \\
\hline Amer & $2,3^{b}$ & $1,9^{\mathrm{b}, \mathrm{c}}$ & $3,3^{\mathrm{a}}$ & $1,5 \mathrm{c}$ & NS & $* * *$ & $* *$ \\
\hline \multicolumn{8}{|l|}{ Texture } \\
\hline Collant & $4,6^{\mathrm{a}}$ & $4,3^{\mathrm{a}}$ & $1,8^{\mathrm{b}}$ & $2,1^{\mathrm{b}}$ & $* * *$ & NS & NS \\
\hline Pâteux & $3,6^{\mathrm{a}}$ & $3,6^{\mathrm{a}}$ & $2,5^{\mathrm{b}}$ & $2,5^{\mathrm{b}}$ & $* * *$ & NS & NS \\
\hline Granuleux & $0,7^{\mathrm{c}}$ & $1,3^{\mathrm{b}}$ & $3,9^{\mathrm{a}}$ & $3,8^{\mathrm{a}}$ & $* * *$ & NS & * \\
\hline
\end{tabular}

Abréviations : voir tableau 1 .

Abbreviations: see table I.

tique présente peu de spécificité, que ce soit au démoulage ou pendant l'affinage.

Les teneurs plus fortes d'AGL minoritaires dans les fromages à matière grasse de chèvre (PP et PM) s'expliquent par leur présence en quantités supérieures (sauf le C5:0) dans les triglycérides de chèvre que dans ceux de vache $[16,23]$. Dans les PP, l'influence de la fraction écrémée de vache sur ces composés, ainsi que sur les esters, les méthyl-cétones et les alcools secondaires, tous issus des acides gras, est sans doute liée à l'intensité de l'acidité grasse de cette fraction. Si, par contre, dans les PM, il n'est pas possible de préciser le rôle respectif de chaque fraction sur la composition en volatils, cela est sans doute dû aux activités de dégradation de la flore de surface qui ont pu niveler les différences dues au type de lait.

\subsection{Flaveur}

Parmi les acides gras étudiés, six présentent un intérêt particulier, car ils ont été décrits comme ayant un arôme « chèvre » $[4$, 23, 24]. Il s'agit des C6:0, C8:0 et C10:0 pour les acides gras majoritaires, des 4MeC8:0, 4EtC8:0 et C9:0 pour les acides gras minoritaires. Ces acides gras semblent contribuer à l'arôme chèvre des fromages. En effet, mis à part le C9:0 et le 4EtC8:0 (absent) dans les PP, ils sont tous trouvés en quantités plus importantes dans les fro- 
Tableau VI. Caractéristiques sensorielles des fromages à pâte molle en fin d'affinage. Moyennes des quatre fabrications pour chaque type de fromage.

Table VI. Sensory characteristics of soft cheeses at the end of ripening. Means of four replicates for each type of cheese.

\begin{tabular}{|c|c|c|c|c|c|c|c|}
\hline & VV & VC & $\mathrm{CV}$ & $\mathrm{CC}$ & LE & MG & $\mathrm{LE} \times \mathrm{MG}$ \\
\hline \multicolumn{8}{|l|}{ Odeur } \\
\hline Chèvre & $1,9^{b}$ & $3,3^{\mathrm{a}}$ & $3,2^{\mathrm{a}}$ & $3,8^{\mathrm{a}}$ & $* * *$ & $* * *$ & NS \\
\hline Lait frais & $1,7^{\mathrm{a}}$ & $1,0^{\mathrm{b}}$ & $1,1^{\mathrm{b}}$ & $0,4^{c}$ & $* * *$ & $* * *$ & NS \\
\hline Lait acidifié & 1,6 & 1,8 & 1,3 & 1,7 & NS & NS & NS \\
\hline Beurre & $1,5^{\mathrm{a}}$ & $0,6^{\mathrm{b}}$ & $1,2^{\mathrm{a}, \mathrm{b}}$ & $0,7^{b}$ & NS & $* * *$ & NS \\
\hline Piquant & 1,3 & 1,7 & 1,7 & 2,0 & NS & NS & NS \\
\hline Intensité & 4,5 & 4,4 & 4,1 & 5,1 & NS & NS & NS \\
\hline \multicolumn{8}{|l|}{ Arôme } \\
\hline Chèvre & $2,0^{\mathrm{b}}$ & $2,8^{\mathrm{a}, \mathrm{b}}$ & $2,6^{\mathrm{a}, \mathrm{b}}$ & $3,5^{\mathrm{a}}$ & * & $* *$ & NS \\
\hline Persistance chèvre & $1,2^{b}$ & $1,7^{b}$ & $1,5^{b}$ & $2,5^{\mathrm{a}}$ & $*$ & $* *$ & NS \\
\hline Piquant & 2,2 & 1,8 & 2,7 & 2,3 & $*$ & NS & NS \\
\hline Persistance piquant & 1,6 & 1,3 & 2,1 & 1,7 & $*$ & NS & NS \\
\hline Lait acidifié & 0,5 & 0,2 & 0,6 & 0,6 & NS & NS & NS \\
\hline Rance & 1,2 & 0,9 & 1,2 & 0,7 & NS & $*$ & NS \\
\hline Intensité & 5,2 & 5,0 & 5,3 & 5,1 & NS & NS & NS \\
\hline \multicolumn{8}{|l|}{ Goût } \\
\hline Salé & 4,2 & 4,0 & 4,1 & 4,1 & NS & NS & NS \\
\hline Acide & $2,7^{\mathrm{a}, \mathrm{b}}$ & $2,6^{b}$ & $3,3^{\mathrm{a}}$ & $3,0^{a, b}$ & $* *$ & NS & NS \\
\hline Amer & $3,6^{\mathrm{a}}$ & $2,5^{b}$ & $1,1^{c}$ & $1,2^{c}$ & $* * *$ & NS & NS \\
\hline \multicolumn{8}{|l|}{ Texture } \\
\hline Collant & $4,9^{b}$ & $5,2^{a, b}$ & $5,2^{\mathrm{a}, \mathrm{b}}$ & $5,6^{\mathrm{a}}$ & NS & NS & NS \\
\hline Pâteux & 4,0 & 4,3 & 4,5 & 4,8 & $*$ & NS & NS \\
\hline Granuleux & 0,9 & 0,6 & 0,7 & 0,7 & NS & NS & NS \\
\hline $\mathrm{Sec}$ & $2,8^{\mathrm{b}}$ & $3,4^{b}$ & $3,3^{\mathrm{b}}$ & $4,1^{\mathrm{a}}$ & $* *$ & NS & NS \\
\hline Ferme & $0,9^{\mathrm{b}}$ & $1,4^{\mathrm{a}}$ & $1,1^{a, b}$ & $1,5^{\mathrm{a}}$ & NS & $* *$ & NS \\
\hline Fondant & 6,3 & 6,0 & 6,0 & 5,8 & NS & $* *$ & NS \\
\hline
\end{tabular}

Abréviations : voir tableau I.

Abbreviations: see table I.

mages à flaveur « chèvre » la plus forte : fromages à matière grasse de chèvre pour les PP, fromages pur chèvre pour les PM. Un résultat similaire a déjà été observé par Pierre et al. [30] dans une étude intra-espèce sur des PM. Il est par ailleurs intéressant de noter que l'influence de chaque fraction sur l'intensité de la flaveur « chèvre » des fromages est différente selon la technologie. Dans les PP, l'odeur et l'arôme « chèvre » sont déterminés uniquement par la présence de la matière grasse de chèvre. Dans les PM, en revanche, la fraction écrémée de chèvre intervient également, ce qui peut être dû à l'influence de cette fraction, dans ces fromages, sur les quantités en C6:0, C8:0 et $\mathrm{C} 10: 0$, à la présence de $4 \mathrm{MeC} 8: 0$ ou 4EtC8:0 libres en quantités supérieures au seuil de détection dans la fraction écrémée, ou à la présence de la flore de surface.

Alors que les précédentes études intraespèces $[19,29]$ ont montré que la flaveur « chèvre » était corrélée à l'acidité grasse des fromages, ce n'est pas le cas dans cette étude, où elle est plus forte avec le lait de vache. Cela signifie que, si la flaveur « chèvre » est liée à la libération d'acides gras, la composition de la matière grasse 
prime devant l'intensité de la lipolyse. Cela confirme l'importance des acides gras minoritaires plus ou moins spécifiques de la matière grasse de chèvre, tels que les $4 \mathrm{MeC} 8: 0$ et $4 \mathrm{EtC} 8: 0$, sur la flaveur « chèvre » des fromages. Par ailleurs, l'influence de la fraction protéique sur la flaveur « chèvre » de fromages à PP observée par Lamberet et al. [19] dans une étude intra-espèce n'est pas retrouvée ici, dans la comparaison de deux espèces différentes.

En ce qui concerne l'intensité globale de l'arôme, plus forte dans les PM $(5,0-5,3)$ que dans les PP $(3,44,5)$, elle peut être mise en relation avec l'intensité de la protéolyse et la présence de composés volatils, plus fortes dans les PM que dans les PP. En revanche, pour une même technologie, il n'apparaît pas de relation entre l'intensité globale de l'odeur et de l'arôme des fromages et les processus biochimiques observés. À l'exception de l'acidité dans les PM, les autres sensations olfacto-gustatives sont généralement plus intenses avec le lait écrémé ou la crème de vache dans les deux technologies. Ainsi, les sensations de « rance » et de « piquant » sont sans doute liées à la teneur en certains AGL des fromages $[4,33]$. En revanche, il est vraisemblable que les odeurs de «beurre » et de « lait frais » soient plutôt dues à des molécules spécifiques de l'espèce bovine.

\subsection{Texture}

Pour les PP, il ressort une nette influence de l'origine de la fraction écrémée sur les caractéristiques de texture, ce qui a précédemment été observé par Lamberet et al. [19]. Mais il est difficile ici d'en connaître la cause, car les différences peuvent provenir de plusieurs phénomènes, qui varient simultanément dans ces fromages. En effet, au type de fraction écrémée se trouvent associées des différences de structure du réseau protéique, liées aux différences de compositions initiales des caséines, des différences dans le degré de protéolyse, et des diffé- rences de $\mathrm{pH}$. D'après la revue de Lawrence et al. [20], ces trois facteurs ont tous une influence majeure sur la texture des fromages. Dans les PM, l'influence respective de la fraction écrémée et de la fraction grasse sur les caractéristiques de texture est difficile à mettre en évidence, puisque pour tous les descripteurs, les fromages pur chèvre et pur vache présentent des notes extrêmes. Cependant, le caractère plus ferme des fromages à matière grasse de chèvre est sans doute une conséquence de la teneur en extrait sec plus forte de ces fromages [20]. L'hétérogénéité de la composition physico-chimique des fromages rend donc ici difficile la détermination de l'influence de chaque fraction sur leur texture, dans les deux technologies étudiées.

\section{REMERCIEMENTS}

Ce travail a été financé par l'Inra, dans le cadre d'une Action incitative programmée portant sur la maturation des produits alimentaires - lait de chèvre.

Nous remercions D. Lefier, M.H. Duployer et $\mathrm{D}$. Demaizières pour leur collaboration technique.

\section{RÉFÉRENCES}

[1] Adda J., Les mécanismes de formation de la flaveur dans les fromages, in : XXII Int Dairy Congress, The Hague, Netherlands, 1986, pp. 169-177.

[2] AFNOR, Analyse sensorielle. Méthodologie : recherche de descripteurs pour l'élaboration d'un profil sensoriel. NF V 09-021 (1990).

[3] Banks J.M., Muir D.D., McNulty D., Dreyer I., Sensory properties of Cheddar type cheese produced from recombined milk fat and casein fractions of bovine and ovine origin, Int. J. Dairy Technol. 50 (1997) 73-78.

[4] Brennand C.P., Ha J.K., Lindsay R.C., Aroma properties and threshold of some branched-chain and other minor volatile fatty acids occuring in milk fat and meat lipids, J. Sens, Stud, 4 (1989) 105-120.

[5] Dagnélie P., Test de Newman et Keuls, in : Théorie et méthodes statistiques, 2, Presses agronomiques de Gembloux, 1975, pp. 245-250. 
[6] Decaen C., Adda J., Évolution de la sécrétion des acides gras des matières grasses du lait au cours de la lactation de la vache, Ann. Biol. Anim. Biochim. Biophys. 10 (1970) 659-677.

[7] Deeth H.C., Fitz-Gerald C.H., Snow A.J., A gas chromatographic method for the quantitative determination of free fatty acids in milk and milk products, N.Z. J. Dairy Sci. 18 (1983) 13-20.

[8] Delacroix-Buchet A., Degas C., Lamberet G., Vassal L., Influence des variants AA et FF de la caséine $\alpha$, caprine sur le rendement fromager et les caractéristiques sensorielles des fromages, Lait 76 ( 1996) 217-241.

[9] Dumont J.P., Adda J., Isolement des constituants de l'arôme des fromages : comparaison des méthodes, Lait 515-516 (1972) 311-323.

[10] FIL, Fromages et fromages fondus. Détermination de l'extrait sec total : méthode de référence. Norme FIL-IDF 4A, Bruxelles (1982).

[11] FIL, Fromages et fromages fondus. Détermination de la teneur en chlorures: méthode par dosage potentiométrique, Norme FIL-IDF 88A, Bruxelles (1988).

[12] FIL, Determination of individual free fatty acids - reference method 265 (1991) 41-44.

[13] Grappin R., Rank T.C.. Olson N.F., Primary proteolysis of cheese proteins during ripening. A review, J. Dairy Sci. 68 (1985) 531-540.

[14] Gripon J.C., Mould-ripened cheeses, in: Fox P.F. (Ed.), Cheese: chemistry, physics and microbiology. 2. Major cheese groups, Elsevier applied science, London, 1987, pp. 121-150.

[15] Gripon J.C.. Desmazeaud M., Le Bars D., Bergère J.L., Étude du rôle des microorganismes et des enzymes au cours de la maturation des fromages, II. Influence de la présure commerciale, Lait 55 (1975) 502-516.

[16] Ha J.K., Lindsay R.C., Release of volatile branched-chain and other fatty acids from ruminant milk fats by various lipases, J. Dairy Sci. 76 (1993) 677-690.

[17] Heil F., Dumont J.P., Caractéristiques organoleptiques de fromages de chèvre fabriqués à partir de laits contenant des variants génétiques différents de la caséine $\alpha_{s l}$, Lait 73 (1993) 559-565.

[18] Heiss E., Essais de dosage de la matière grasse dans le fromage par des méthodes rapides, Deut, Milch Zeit, 82 (1961) 3.

[19] Lamberet G., Degas C., Delacroix-Buchet A., Vassal L., Influence de caractères liếs aux allèles A et $F$ de la caséine $\alpha_{\mathrm{s} 1}$ caprine sur la flaveur chèvre : fabrications fromagères avec échange de protéines et de matières grasses, Lait 76 (1996) 349-361.

[20] Lawrence R.C., Creamer L.K., Gilles J., Texture development during cheese ripening, J. Dairy Sci. 70 (1987) 1748-1760.

[2I] Le Mens P., Le lait de chèvre : propriétés physico-chimiques, nutritionnelles et chimiques. in : Luquet F.M. (éd.), Laits et produits laitiers, vache, brebis, chèvre. 1. Les laits, de la mamelle à la laiterie. Tec et doc Lavoisier, Apria Paris. 1985 , pp. 349-368.

[22] Lenoir J., Gripon J.C., Lamberet G., Cerning J., Les Penicillium, in : Hermier J., Lenoir J., Weber F. (éd.), Les groupes microbiens d'intérêt laitier. CEPIL, Paris, 1992, pp. 221-257.

[23] Le Quếré J.L., Pierre A., Riaublanc A., Demaizières D., Characterization of aroma compounds in the volatile fraction of soft goat cheese during ripening, Lait 78 (1998) 279-290.

[24] Le Quéré J.L., Septier C., Demaizières D., Salles C., Identification and sensory evaluation of the character-impact compounds of goat cheese flavour, in: Taylor A.J., Mottram D.S. (Eds.), Flavour science, recent developments, 8 th Weurman flavour research symposium. Reading, GB, 1996, pp. 325-330.

[25] Mac Fie H., Bratchell N., Greenhoff K., Vallis L.V., Designs to balance the effect of order of presentation and first-order carry-over effects in Hall tests, J. Sens. Stud. 4 (1989) 129-148.

[26] Marshall R.J., Combined instrumental and sensory measurement of the role of fat in food texture, Food Qual. Preference 2 (1991) 117-124.

[27] Needs E.C., Ford G.D., Owen A.J., Tuckley B., Anderson M., A method for the quantitative determination of individual free fatty acids in milk by ion exchange resin adsorption and gasliquid chromatography, J. Dairy Res. 50 (1983) 321-329.

[28] Pearce K.N., The complexometric determination of calcium in dairy products, N. Z. J. Dairy Sci. Technol. 12 (1977) 113-115.

[29] Pierre A., Le Quéré J.L., Famelart M.H., Riaublanc A., Rousseau F, Composition, yields, texture and aroma compounds of goat cheeses as related to the $A$ and $O$ variants of $\alpha_{s 1}$ casein in milk, Lait 78 (1998) 291-301.

[30] Pierre A., Le Quéré J.L., Riaublanc A., Le Graët Y., Demaizières D.. Michel F., Composition and physico-chemical characteristics of goat milks containing the $\mathrm{A}$ or $\mathrm{O} \alpha_{\mathrm{s} 1}$ casein variants, Lait 78 (1998) 191-202.

[3I] Spinnler H.E., Guichard E., Gripon J.C., La flaveur des fromages, in : Eck A., Gillis J.C. (éd.), Le fromage, Tec et doc Lavoisier, Paris, 1997. pp. 493-505.

[32] Vassal L., Delacroix-Buchet A., Bouillon J., Influence des variants AA, EE et FF de la caséine $\alpha_{\mathrm{s} 1}$ caprine sur le rendement fromager et les caractéristiques sensorielles de fromages traditionnels : premières observations, Lait 74 (1994) 89-103.

[33] Woo A.H., Lindsay R.C., Statistical correlation of quantitative flavor intensity assessments and individual free fatty acid measurements for routine detection and prediction of hydrolytic rancidity off-flavors in butter, J. Food Sci, 48 (1983) 1761-1771. 